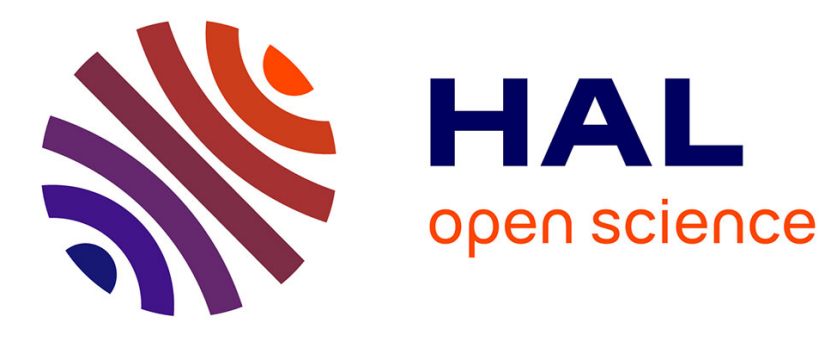

\title{
Perfect Brewster transmission through ultrathin perforated films
}

Kim Pham, Agnès Maurel, Jean-François Mercier, Simon Félix, Maria Luisa Cordero, Camila Horvath

\section{- To cite this version:}

Kim Pham, Agnès Maurel, Jean-François Mercier, Simon Félix, Maria Luisa Cordero, et al.. Perfect Brewster transmission through ultrathin perforated films. Wave Motion, 2020, 93, pp.102485. 10.1016/j.wavemoti.2019.102485 . hal-03089421

\section{HAL Id: hal-03089421 https://hal.science/hal-03089421}

Submitted on 8 Jan 2021

HAL is a multi-disciplinary open access archive for the deposit and dissemination of scientific research documents, whether they are published or not. The documents may come from teaching and research institutions in France or abroad, or from public or private research centers.
L'archive ouverte pluridisciplinaire HAL, est destinée au dépôt et à la diffusion de documents scientifiques de niveau recherche, publiés ou non, émanant des établissements d'enseignement et de recherche français ou étrangers, des laboratoires publics ou privés. 


\title{
Perfect Brewster transmission through ultrathin perforated films
}

\author{
K. Pham ${ }^{1}$, A. Maurel' ${ }^{2}$, J.-F. Mercier ${ }^{3}$, S. Félix ${ }^{4}$, M.L. Cordero ${ }^{5}$ and C. \\ Horvath $^{5}$ \\ ${ }^{1}$ IMSIA, ENSTA ParisTech-CNRS - EDF - CEA, Université Paris-Saclay, 828 Bd des \\ Maréchaux, 91732 Palaiseau, France. \\ 2 Institut Langevin, ESPCI ParisTech, CNRS UMR 7587, 1 rue Jussieu, 75005 Paris, \\ France. \\ ${ }^{3}$ Poems, CNRS, ENSTA ParisTech, INRIA, 828 Bd des Maréchaux, 91762 Palaiseau, \\ France. \\ ${ }^{4}$ LAUM, CNRS UMR 6613, Le Mans Université, av. Olivier Messiaen, 72085 Le Mans, \\ France. \\ ${ }^{5}$ Departamento de Física Facultad de Ciencias Físicas y Matematicas, Universidad de \\ Chile, Av. Blanco Encalada 2008, Santiago, Chile.
}

\begin{abstract}
We address the perfect transmission of a plane acoustic wave at oblique incidence on a perforated, sound penetrable or rigid, film in two-dimensions. It is shown that the Brewster incidence $\theta^{*}$ realizing so-called extraordinary transmission due to matched impedances varies significantly when the thickness $e$ of the film decreases. For thick films, i.e. $k e \gg 1$ with $k$ the incident wavenumber, the classical effective medium model provides an accurate prediction of the Brewster angle $\theta^{*}=\theta_{\mathrm{B}}$ independent of $e$. However, for thiner and thiner films with $k e<1$, $\theta^{*}$ becomes dependent of $e$ and it deviates from $\theta_{\mathrm{B}}$. To properly describe this shift, an interface model is used which accurately reproduces the spectra of ultrathin to relatively thick perforated films. Depending on the contrasts in the material properties of the film and of the surrounding matrix, decreasing the film thickness can produce an increase or a decrease of $\theta^{*}$; it can also produce the disappearance of a perfect transmission or to the contrary its appearance.
\end{abstract}

\section{Introduction}

Extraordinary optical/acoustical transmission (EOT/EAT) refers to high transmission through films with subwavelength apertures (figure 1), while a single aperture would transmit light/sound very poorly [1. Such high transmission is made possible thanks to collective effects of the holes. Collective effects can render the perforated film able to support surface waves, as observed in plasmonic structures [2] or able to support resonances of the Fabry-Perot type 


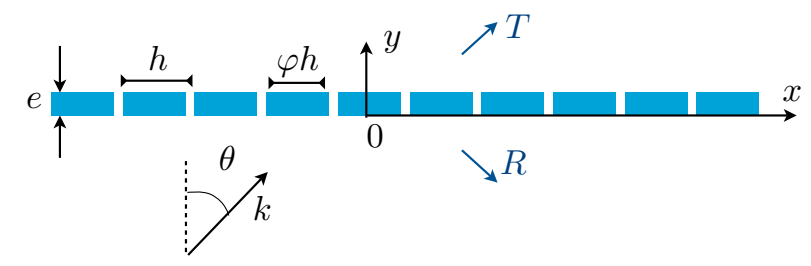

Figure 1: Scattering of an acoustic wave at oblique incidence $\theta$ on a perforated film. The mass densities and sound velocities are $\left(\rho_{\mathrm{i}}, c_{\mathrm{i}}\right)$ in the film material and $\left(\rho_{\mathrm{m}}, c_{\mathrm{m}}\right)$ in the matrix; $k=\omega / c_{\mathrm{m}}$ is the wavenumber in the matrix.

[3, 4]. But they can also be non resonant when they lend to the film an effective impedance which matches that of the surrounding matrix at the so-called Brewster incidence [5, 6] and, in contrast with resonance-based mechanisms, the resulting EOT has the advantage to be mildly affected by the losses [14. It has been shown that the Brewster incidence can be tuned by playing with the geometrical parameters of perforated rigid screens [7, 8] and this has been used to realize beam shifter [9] and flat lenses [10]; similar tuning has been obtained by playing on the material properties of sound penetrable films [11, 12, 13.

From a theoretical point of view, perfect transmissions have been analyzed owing to effective medium theories based on classical homogenization. With $h$ the spacing and $k$ the incident wavenumber, classical homogenization predicts that for $k h \ll 1$ the perforated film can be replaced by an equivalent homogeneous one for which the scattering properties are explicitly known. Such approaches have been applied firstly to sound-rigid films in acoustics [15, 16, and their electromagnetic counterparts [5, 17] and they have been then generalized to more involved geometric perforations [7, 8, and to sound-penetrable films [11, 12. By construction, effective medium theories aim to describe the effective propagation within the film and they disregard the evanescent field excited at its end boundaries. This is intuitively justified if the film is thick enough to ensure that the effects of wave propagation are dominant compared to that of the boundary layers due to the evanescent field. When the boundary layer effects cannot be neglected, other strategies of homogenization have to be sought. This may happens when they are as important as the effects of wave propagation [18, 19] or when they become dominant [20, 21, 22]. In the present case, we expect that the boundary layer effects will become dominant when $k e \ll 1$. In this limit, we already know that perfect transmission based on Fabry-Perot resonances cannot take place (they require $k e>\pi$ ). The aim of the present study is to characterize the perfect transmissions based on matched impedances for thin $k e \sim 1$ and ultrathin $k e \ll 1$ films. To do so, we use an effective interface model 22 which provides the condition of existence of perfect transmissions and when perfect transmission is possible, a prediction for $\theta^{*}$.

The paper is organized as follows. In the Section 2, the problem is set and typical variations of the Brewster angles with the film thickness are given. 
In Section 3 the classical effective medium model is recalled, resulting in a prediction of the Brewster incidence $\theta^{*}=\theta_{\mathrm{B}}$, Eq. (8) valid for thick films $k e>1$. We also provide the predictions given by the effective interface model detailed in 22] and the resulting Brewster angle $\theta^{*}$, (13), valid for thin films $k e<1$. The variations of $\theta^{*}$ when going from thin to thick films are inspected and validated with direct numerics in Section 4 . We collect in the appendices additional calculations and results.

\section{Perfect transmissions through perforated films}

We consider propagation of acoustic waves in the harmonic regime with time dependence $e^{-i \omega t}$. The propagation is described by the wave equation for the pressure $p$ of the form

$$
\operatorname{div}\left(\frac{1}{\rho(\mathbf{x})} \boldsymbol{\nabla} p\right)+\omega^{2} \chi(\mathbf{x}) p=0,
$$

with the mass density $\rho(\mathbf{x})$ and the isentropic compressibility $\chi(\mathbf{x})$ varying in space $\mathbf{x}=(x, y)$, see figure 1. In the perforated film, with thickness $e$ and filling fraction $\varphi$, they are denoted $\rho_{\mathrm{i}}$ and $\chi_{\mathrm{i}}=1 /\left(\rho_{\mathrm{i}} c_{\mathrm{i}}^{2}\right)$, with $c_{\mathrm{i}}$ the sound speed; in the surrounding matrix, they are denoted $\rho_{\mathrm{m}}$ and $\chi_{\mathrm{m}}=1 /\left(\rho_{\mathrm{m}} c_{\mathrm{m}}^{2}\right)$. With $k=\omega / c_{\mathrm{m}}$ the incident wavenumber, the wave propagation in the matrix reads

$$
\Delta p+k^{2} p=0, \quad y \notin(0, e) .
$$

This problem can be solved numerically to get the scattering coefficients $(R, T)$ for a plane wave at oblique incidence $\theta$ on the film. The numerical results presented in the paper are obtained using a multimodal method for sound-hard films 21 and adapted for penetrable ones (see Appendix A). We report in figure 2 typical reflection spectra for a sound rigid film and for three penetrable films against the incidence $\theta$ when varying the thickness of the film $k e \in(0,1)$ for $k h=1$; perfect transmissions are visible by means of zero reflections $|R|=0$. In the panels (a) and (b), a perfect transmission takes place for each value of $e$ at an incidence $\theta^{*}$ being dependent on $e$. Starting for vanishing thickness with $\theta^{*}=0$ in panel (a) and with $\theta^{*}=90^{\circ}$ in panel (b), the Brewster incidence varies smoothly for increasing $e \in(0, h)$ and eventually reaches a constant value $\theta^{*} \rightarrow \theta_{\mathrm{B}}$ for thicker films. The spectra on the panels (c) and (d) show that the occurence of a perfect transmission is not always possible. In panel (c), perfect transmissions are observed for thin films only, with $\theta^{*}$ experiencing large variations from 0 to $90^{\circ}$ when $e \in(0,0.65 h)$. Reversely, in panel (d), perfect transmissions become possible for $e / h>0.45$ where $\theta^{*}=90^{\circ}$ then $\theta^{*}$ decreases for increasing $e / h$. Incidentally, a noticeable difference between the penetrable films and the sound-rigid one is that a penetrable film with vanishing thickness always produces perfect transmission since in this limit, the film simply disappears. This is not the case for a rigid film: a rigid film with vanishing thickness is a rigid perforated plate able to scatter efficiency the wave. This is why $|R|=0$ for $k e=0$ except on the panel (b). 

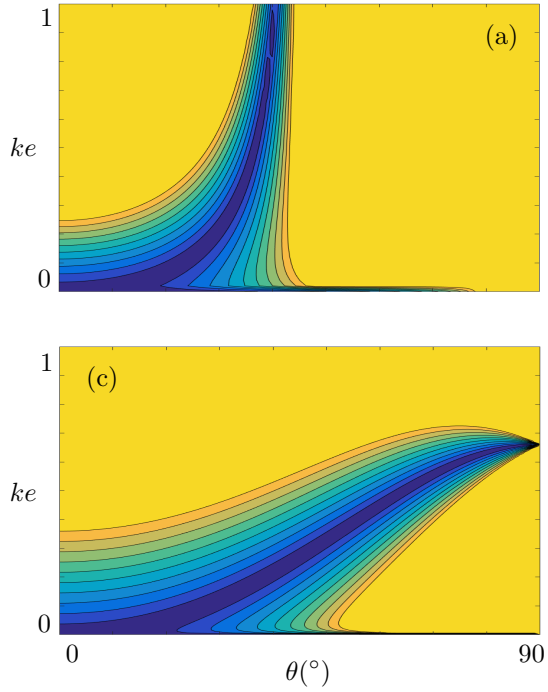

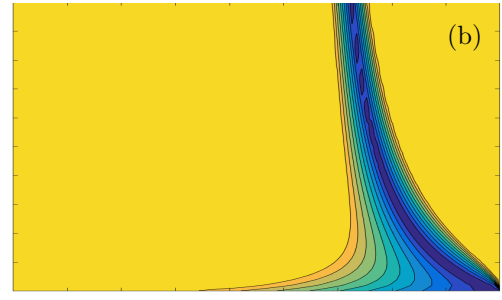

0.1

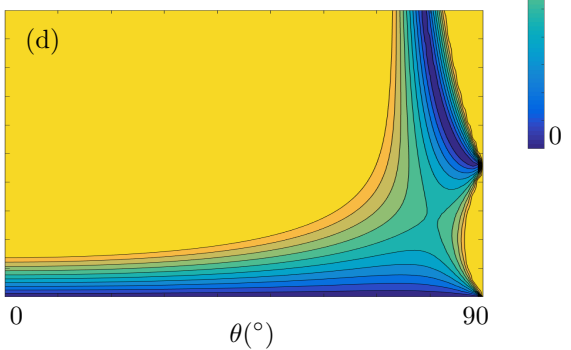

Figure 2: Perfect transmission at the Brewster angle. Reflection $|R|$ computed numerically (in colorscale with saturation for $|R|>0.1$ ) against the incidence $\theta$ and the film thickness $k e$ $(\varphi=0.5$ and $k h=1)$ (a) with $\rho_{\mathrm{i}} / \rho_{\mathrm{m}}=10^{-3}$ and $\chi_{\mathrm{i}} / \chi_{\mathrm{m}}=1$, (b) for a sound-rigid perforated film, (c) with $\rho_{\mathrm{i}} / \rho_{\mathrm{m}}=10^{-3}$ and $\chi_{\mathrm{i}} / \chi_{\mathrm{m}}=10$ and (d) $\rho_{\mathrm{i}} / \rho_{\mathrm{m}}=3.5$ and $\chi_{\mathrm{i}} / \chi_{\mathrm{m}}=3.10^{-7}$.

From these observations, it is not straightforward to anticipate the scattering properties of a perforated film; it is the goal of this study to provide a simplified but still accurate model to predict the existence of perfect transmissions and when they can take place, the corresponding incidence $\theta^{*}$.

\section{Effective medium model and effective interface model}

Effective models aim at simplifying the problem in the scattering region $y \in(0, e)$. In the classical effective medium model, this is done by replacing the perforated film by a homogeneous and anisotropic one; in the effective interface model, this is done by imposing non intuitive jump conditions between $y=0$ and $y=e$. Both models are based on asymptotic homogenization, hence they are valid in the limit $k h \ll 1$ (and we shall see the predictions hold up to $k h \sim 1$ with reasonable errors). However, while the effective medium model is valid for thick films $k e \gg 1$, the effective interface model is valid in the opposite limit of thin films $k e \ll 1$.

\subsection{Classical effective medium model}

The classical model is based on effective medium theory (or classical asymptotic homogenization); it tells us that a medium made of layers can be replaced by an equivalent homogeneous and anisotropic medium described by the wave 
equation

$$
\operatorname{div}\left(\left(\begin{array}{ll}
\rho_{\mathrm{m}} / \rho_{x} & 0 \\
0 & \rho_{\mathrm{m}} / \rho_{y}
\end{array}\right) \nabla p\right)+\chi_{\mathrm{r}} k^{2} p=0, \quad y \in(0, e),
$$

involving effective mass densities $\left(\rho_{x}, \rho_{y}\right)$ and a relative effective compressibility $\chi_{\mathrm{r}}$ defined by

$$
\begin{cases}\frac{\rho_{\mathrm{m}}}{\rho_{y}}=\varphi \frac{\rho_{\mathrm{m}}}{\rho_{\mathrm{i}}}+1-\varphi, & \frac{\rho_{x}}{\rho_{\mathrm{m}}}=\varphi \frac{\rho_{\mathrm{i}}}{\rho_{\mathrm{m}}}+1-\varphi \\ \chi_{\mathrm{r}}=\varphi \frac{\chi_{\mathrm{i}}}{\chi_{\mathrm{m}}}+(1-\varphi) . & \end{cases}
$$

Within this description, the perforated film is replaced by a homogeneous one of same thickness $e$ where (3) applies surrounded by the matrix where (2) applies. It follows that for an incident plane wave at incidence $\theta$, the solution reads

$$
p(\mathbf{x})=e^{i k x \sin \theta} \begin{cases}\left(e^{i k y \cos \theta}+R e^{-i k y \cos \theta}\right), & y \in(-\infty, 0), \\ \left(A e^{i K y}+B e^{-i K y}\right), & y \in(0, e), \\ T e^{i k(y-e) \cos \theta}, & y \in(e,+\infty) .\end{cases}
$$

At $y=0, e$, by using the continuities of the pressure and of the normal velocity $u_{y}\left(u_{y}=\frac{1}{\rho_{y}} \partial_{y} p\right.$ in the effective film and $u_{y}=\frac{1}{\rho_{\mathrm{m}}} \partial_{y} p$ in the matrix), we get the scattering coefficients of the form

$$
\left\{\begin{array}{l}
R=\frac{2 i\left(\xi^{2}-1\right) \sin K e}{(\xi-1)^{2} e^{i K e}-(\xi+1)^{2} e^{-i K e}}, \\
T=-\frac{4 \xi}{(\xi-1)^{2} e^{i K e}-(\xi+1)^{2} e^{-i K e}},
\end{array}\right.
$$

with

$$
K=k \sqrt{\frac{\rho_{y}}{\rho_{\mathrm{m}}} \chi_{\mathrm{r}}-\frac{\rho_{y}}{\rho_{x}} \sin ^{2} \theta}, \quad \xi=\frac{\rho_{y}}{\rho_{\mathrm{m}}} \frac{k \cos \theta}{K} .
$$

The Brewster incidence $\theta_{\mathrm{B}}$ realizing perfect transmission independently of the frequency $k$ corresponds to the condition of impedance matching, $\xi=1$, which provides the relation

$$
\cos \theta_{\mathrm{B}}=\sqrt{\frac{\rho_{\mathrm{m}}-\chi_{\mathrm{r}} \rho_{x}}{\rho_{\mathrm{m}}-\rho_{x} \rho_{y} / \rho_{\mathrm{m}}}} .
$$

Eventually, perfect transmissions due to Fabry-Perot resonances is obtained for $K e=n \pi$ with $n$ integer; it corresponds to constructive interferences during wave propagation within the film and, as previously said, it requires large film thickness $e$. 


\subsection{Effective interface model}

The effective model based on homogenization of thin interface has been detailed in 22. It aims at capturing the boundary layer effects due to the evanescent field excited at the end boundaries of the perforated film. Because of the low frequency regime, the evanescent field ressembles a static one associated with the Laplace equation. This allows us to encapsulate the effect of the evanescent fields in effective, though non intuitive, transmission conditions. These conditions tell us that the pressure and the normal velocity are not continuous but they experience jumps, specifically

$$
\llbracket p \rrbracket=h \mathcal{B} \frac{\partial \bar{p}}{\partial y}, \quad \llbracket \frac{\partial p}{\partial y} \rrbracket=-h \mathcal{C} \frac{\partial^{2} \bar{p}}{\partial x^{2}}-h \mathcal{D} k^{2} \bar{p},
$$

where for any field $f, \llbracket f \rrbracket \equiv f(x, e)-f(x, 0)$ and $\bar{f} \equiv \frac{1}{2}[f(x, e)+f(x, 0)]$. Among the 3 interface parameters $(\mathcal{B}, \mathcal{C}, \mathcal{D})$ entering in the jump conditions, $\mathcal{D}$ is explicitly known and it reads

$$
\mathcal{D}=\chi_{\mathrm{r}} \frac{e}{h},
$$

with $\chi_{\mathrm{r}}$ defined in $(4)$, while $(\mathcal{B}, \mathcal{C})$ are deduced from static problems. In general, $\mathcal{B}$ and $\mathcal{C}$ have to be determined numerically (see Appendix B (B.1) and figure B.10). However, we shall see that close forms are known in the limits of thin and thick films, see forthcoming (14).

From (9), the perforated film has been replaced by a region $y \in(0, e)$ across which jumps apply. It is worth noting that the solution in $y \in(0, e)$ does not need to be specified (and in fact it cannot be specified). The jumps are sufficient

to link the behavior of the wave on both sizes of the interface and doing so, the solution of the scattering problem reads

$$
p(\mathbf{x})=e^{i k x \sin \theta} \begin{cases}e^{i k y \cos \theta}+R e^{-i k y \cos \theta}, & y \in(-\infty, 0), \\ T e^{i k(y-e) \cos \theta}, & y \in(e,+\infty),\end{cases}
$$

with

$$
\left\{\begin{aligned}
R & =\frac{1}{2}\left(\frac{z_{1}}{z_{1}^{*}}-\frac{z_{2}}{z_{2}^{*}}\right), & & T=\frac{1}{2}\left(\frac{z_{1}}{z_{1}^{*}}+\frac{z_{2}}{z_{2}^{*}}\right), \\
z_{1} & =1+\frac{i k h}{2} \mathcal{B} \cos \theta, & z_{2} & =1+\frac{i k h}{2 \cos \theta}\left(\mathcal{D}-\mathcal{C} \sin ^{2} \theta\right) .
\end{aligned}\right.
$$

It follows that perfect transmission is expected at an incidence $\theta^{*}$ satisfying

$$
\cos \theta^{*}=\sqrt{\frac{\mathcal{C}-\mathcal{D}}{\mathcal{C}-\mathcal{B}}}
$$

and we shall see that $\theta^{*}$ in general differs from the classical prediction $\theta_{\mathrm{B}}$ in (8). Expectedly, the effective interface model is unable to predict resonances of the Fabry-Perot type and this is because the film is assumed to be thin, and notably too thin to satisfy a criterion of the quarter wavelength type. 


\section{Variation of the Brewster angle for thin films}

\subsection{The case of sound-penetrable films}

To begin with, we report in figure 3 the spectrum of the reflection computed numerically and the spectra given by the effective medium theory and given by the effective interface theory. The film is that of the figure 2(a) and we extended the range of thickness to $k e \in\left(10^{-1}-10^{2}\right)$ (with $k h=1$ ). As expected, the actual spectrum is well reproduced by the effective interface model for thin films and it is well reproduced by the effective medium model for thick films; in between, the two models are roughly equivalent in accuracy. For the somehow arbitrary division chosen in figure 3, we get the following absolute errors $\mid R_{\text {num }}-$ $R \mid$ (where $R$ in (6) or $(12)$ ) : for $k e \in\left(10^{-1}-1\right.$ ) it is $0.3 \%$ for the interface model and $13 \%$ for the effective medium model, for $k e \in\left(1-10^{1}\right)$ it is $3 \%$ and $5 \%$ and for $k e \in\left(10^{1}-10^{3}\right)$ it is $20 \%$ and $7 \%$.

(a) Numerics

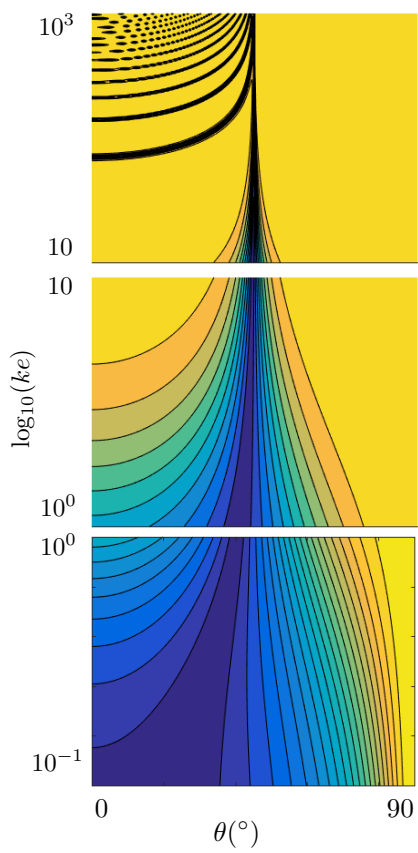

(a) (b) effective medium model
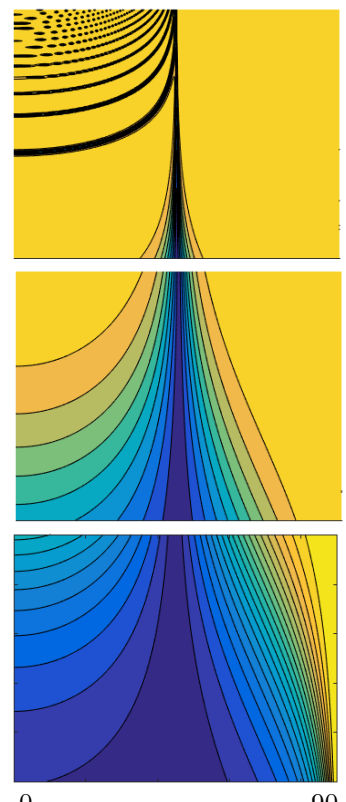

$\theta\left({ }^{\circ}\right)$

(c) effective interface model
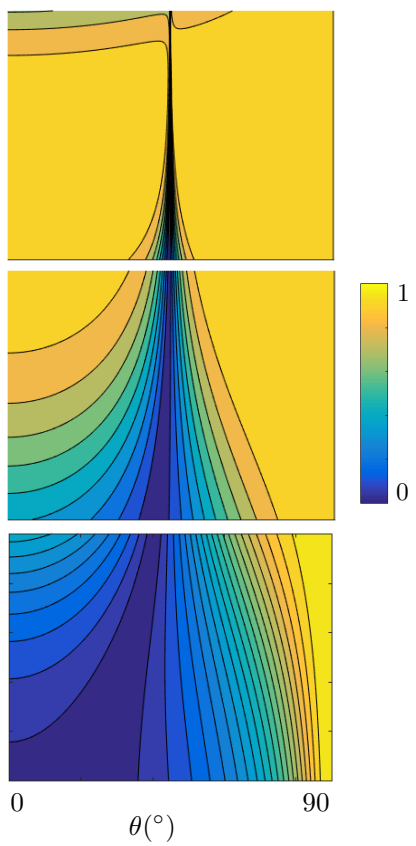

(b)

Figure 3: Reflection spectra against $\theta$ and $\log _{10}(k e)$ for $k h=1\left(\rho_{\mathrm{i}} / \rho_{\mathrm{m}}=10^{-3}\right.$ and $\left.\chi_{\mathrm{r}}=1\right)$. The effective medium model (b) reproduces the actual spectrum (a) for large $k e \in\left(10-10^{3}\right)$ in particular the Fabry-Perot resonances at $K e=n \pi$ in $(7)$; the interface model (c) reproduces the actual spectrum (a) for small $k e \in\left(10^{-1}-1\right)$ in particular the variations of $\theta^{*}$ in $[13$. In the intermediate range $k e \in(1-10)$ the two models are roughly equivalent.

For $k e=0.1$, the profiles are shown in figure 4 (hence $e / h=0.1$ and for this aspect ratio, the interface parameters are $\mathcal{B}=0.037, \mathcal{C}=0.441$ and $\mathcal{D}=0.1$ ); the equivalent interface model is here very accurate while the effective medium 
model clearly fails in a correct prediction. In particular the perfect transmission takes place at $\theta^{*}=20^{\circ}$ well below the usual $\theta_{\mathrm{B}}=40^{\circ}$ prediction. Eventually, the inset shows that the variations of $\theta^{*}$ against $e / h$ are accurately captured by the interface model.

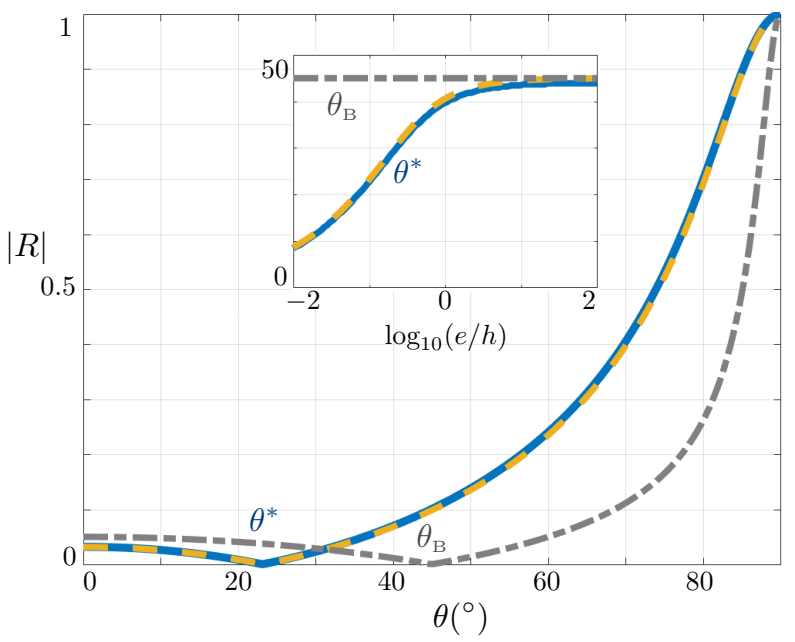

Figure 4: Profile of $|R|$ against $\theta$ from figure 3 for $k e=0.1$. Plain blue line shows $\left|R_{\text {num }}\right|$, dotted yellow lines $|R|$ from the effective interface model $\sqrt{12}$, and dashed grey line from the classical effective medium model (6)- 7). The inset shows the variations of $\theta^{*}$ against $e / h$ ( $k h=0.01$ is used to avoid Fabry-Perot resonances for large $k e$ values).

Similar results, not reported, are found for the the sound-penetrable films considered in the figure $2(\mathrm{c})$ and $(\mathrm{d})$. In each case, $(\mathcal{B}, \mathcal{C})$ are obtained by resolving the static problems (see Appendix B, Eq. (B.1p) and they depend on the characteristics of the film, namely the contrasts $\left(\rho_{\mathrm{i}} / \rho_{\mathrm{m}}, \chi_{\mathrm{i}} / \chi_{\mathrm{m}}\right)$ and the aspect ratio $e / h$; in contrast, they do not depend on the frequency, and this is the gain of the homogenization process. However, a more significant gain would be to have explicit expressions for those parameters, in which case one could anticipate the scattering properties of films and in particular their ability to support perfect transmission. We did not find approximate expressions for $(\mathcal{B}, \mathcal{C})$ valid for any film; however, explicit expressions can be determined in the limits of small and large aspect ratio $e / h$ by inspecting the theoretical bounds of these coefficients. In [22, lower bounds were established and we provide in the Appendix B the derivation of the upper bounds, from which

$$
\frac{\rho_{y}}{\rho_{\mathrm{m}}} \frac{e}{h} \leq \mathcal{B} \leq \frac{\rho_{x}}{\rho_{\mathrm{m}}} \frac{e}{h}, \quad \frac{\rho_{\mathrm{m}}}{\rho_{x}} \frac{e}{h} \leq \mathcal{C} \leq \frac{\rho_{\mathrm{m}}}{\rho_{y}} \frac{e}{h},
$$

where $\left(\rho_{x}, \rho_{y}\right)$ are the geometrical and arithmetical averages of the mass densities defined in (4), and $\rho_{y} \leq \rho_{x}$ by definition. Now, a remarkable fact is that the parameters $(\mathcal{B}, \mathcal{C})$ reach their upper bounds for vanishing thickness $e / h$ and their lower bounds for large $e / h$, as illustrated in figure 5 . 


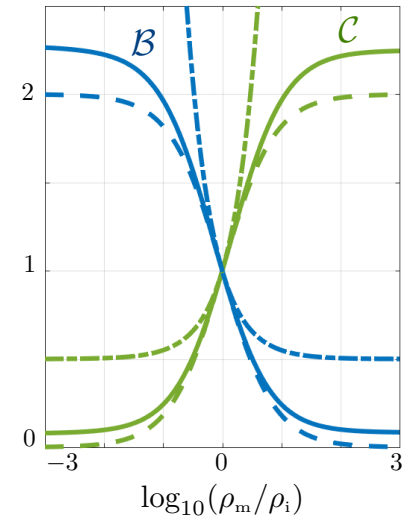

(a)

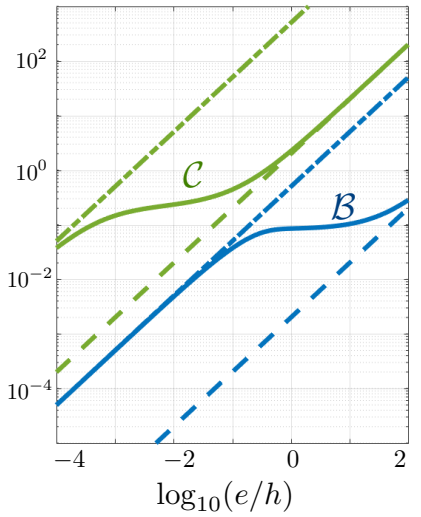

(b)

Figure 5: Interface parameters $\mathcal{B}$ (plain blue line) and $\mathcal{C}$ (plain green line) for $\varphi=0.5$ (a) against $\log _{10}\left(\rho_{\mathrm{m}} / \rho_{\mathrm{i}}\right)$ for $e / h=1$ and (b) against $\log _{10}(e / h)$ for $\rho_{\mathrm{m}} / \rho_{\mathrm{i}}=10^{3}$. In $(\mathrm{a}-\mathrm{b})$, the dashed and the dashed-dotted lines shows the theoretical lower and upper bounds (14.)

Hence, it is sufficient to use the explicit bounds (14) in 13 to get the limiting values of the Brewster angle $\theta^{*}$ namely from the

$$
\text { effective interface model, }\left\{\begin{array}{l}
e / h \ll 1, \quad \cos \theta^{*}=\sqrt{\frac{\rho_{\mathrm{m}}-\chi_{\mathrm{r}} \rho_{y}}{\rho_{\mathrm{m}}-\rho_{x} \rho_{y} / \rho_{\mathrm{m}}}}, \\
e / h \gg 1, \quad \theta^{*}=\theta_{\mathrm{B}},
\end{array}\right.
$$

with $\theta_{\mathrm{B}}$ defined in $(8)$. It is worth noting that in the limit of $e / h \gg 1$, we recover the expression of the Brewster angle $\theta_{\mathrm{B}}$ predicted by the effective medium model; this means that in the limit $k h \ll k e \ll O(1)$ where the effective interface model is valid the Brewster angle reaches $\theta_{\mathrm{B}}$ predicted in the limit $k h \ll O(1) \ll k e$ where the effective medium model is valid. In other words $\theta^{*}$ tends to $\theta_{\mathrm{B}}$ as soon as $e / h \gg 1$ independently of the value of $k e$.

Now, by looking the relations (15) along with (8) we can predict that perfect transmission is not always possible depending on the contrast $\rho_{\mathrm{i}} / \rho_{\mathrm{m}}$ and on $\chi_{\mathrm{r}}$. This is summarised in figure 6 where we report for $\rho_{\mathrm{i}}>\rho_{\mathrm{m}}$ and $\rho_{\mathrm{i}}<\rho_{\mathrm{m}}$ the ranges of $\chi_{\mathrm{r}}$ allowing for perfect transmission in the limits of thin and thick films. For instance when $\rho_{\mathrm{i}} / \rho_{\mathrm{m}}<1$, four scenarios are possible depending on the value of $\chi_{\mathrm{r}}$ : (i) if $\chi_{\mathrm{r}} \in\left(\rho_{y} / \rho_{\mathrm{m}}, \rho_{x} / \rho_{\mathrm{m}}\right)$ perfect transmission is not be observed for thin films but appears for thick films, (ii) if $\chi_{\mathrm{r}} \in\left(\rho_{x} / \rho_{\mathrm{m}}, \rho_{\mathrm{m}} / \rho_{x}\right)$ perfect transmission is always possible and $\theta^{*}$ varies when $e / h$ varies according to the limits in 15), (iii) if $\chi_{\mathrm{r}} \in\left(\rho_{\mathrm{m}} / \rho_{x}, \rho_{\mathrm{m}} / \rho_{y}\right)$, perfect transmission is possible only for thin films; (iv) eventually for $\chi_{\mathrm{r}} \in\left(-\infty, \rho_{y} / \rho_{\mathrm{m}}\right)$ or $\left(\rho_{\mathrm{m}} / \rho_{y},+\infty\right)$, perfect transmission is never realized whatever the film thickness. It is easy to see that two of these scenarios are illustrated in the figures 2(a) and (c). For these films, $\rho_{x} / \rho_{\mathrm{m}} \simeq 0.5, \rho_{y} / \rho_{\mathrm{m}} \simeq 2.10^{-3}$, hence in (a) $\chi_{\mathrm{r}}=1$ results in perfect transmission allowed both for thin and thick films (scenario (ii)) and in (c) $\chi_{\mathrm{r}}=5.5$ results 


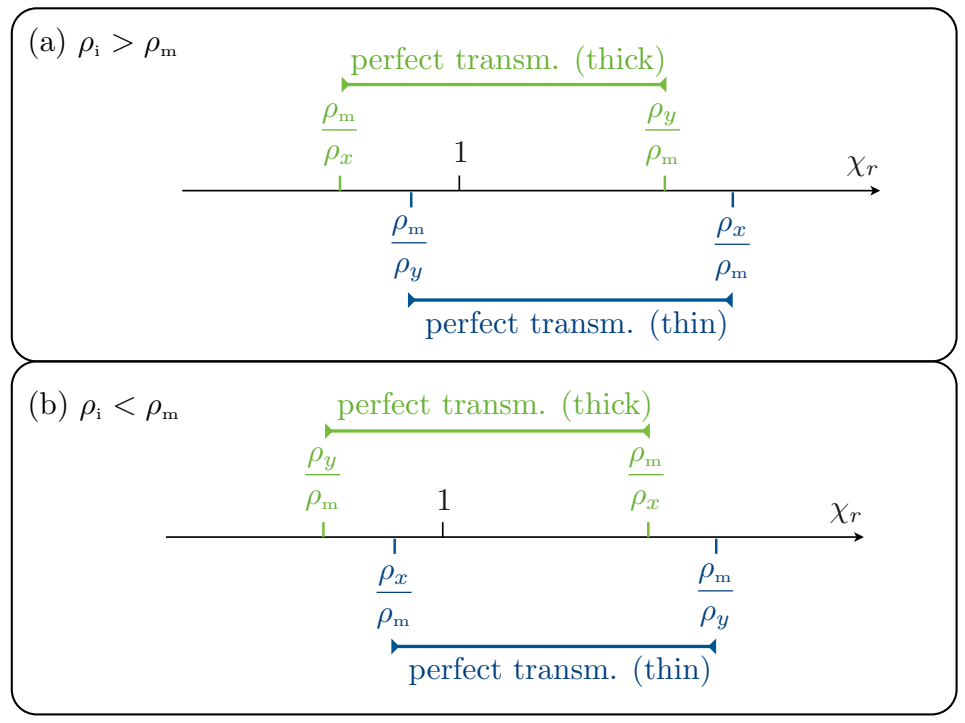

Figure 6: Ranges of parameters for which perfect transmission is allowed in the limits $e / h \ll 1$ and $e / h \gg 1$, from 15 .

in perfect transmission allowed for thin films only (scenario (iii)). Eventually, the case of the figure $2(\mathrm{~d})$ with $\rho_{\mathrm{i}} / \rho_{\mathrm{m}}>1, \rho_{\mathrm{m}} / \rho_{x} \simeq 0.44, \rho_{\mathrm{m}} / \rho_{y} \simeq 0.64$ and $\chi_{\mathrm{r}} \simeq 0.5$ illustrates a case where perfect transmission is not allowed for very thin films. In these 3 cases, the limit of $\theta^{*}$ for vanishing $e / h$ from 15 is for (a) $\theta^{*} \simeq 1.8^{\circ}$, for (c) $\theta^{*} \simeq 5.7^{\circ}$ and for (d) $\theta^{*}$ is found imaginary, in agreement with the observation in figure 2 .

\subsection{The case of sound-hard film}

A case of practical interest in acoustics is that of sound-hard films whose analog in electromagnetism are metallic films for polarized waves in the farinfrared. The effective models have been derived in [21, 23] see also [24], and they correspond to the limit $\rho_{\mathrm{m}} / \rho_{\mathrm{i}}=\chi_{\mathrm{i}} / \chi_{\mathrm{m}}=0$, hence from (4)

$$
\rho_{\mathrm{m}} / \rho_{x}=0, \quad \chi_{\mathrm{r}}=\rho_{\mathrm{m}} / \rho_{y}=(1-\varphi) .
$$

According to the figure 6 (a), this already tells us that a perfect transmission can take place for any value of $e / h$ that is for thin and thick films. But this does not tell much about $\mathcal{B}$ and $\mathcal{C}$ since the bounds in 14 leave us with $\frac{1}{1-\varphi} \frac{e}{h} \leq \mathcal{B} \leq+\infty$ and $0 \leq \mathcal{C} \leq(1-\varphi) \frac{e}{h}$. However, very good estimates of $(\mathcal{B}, \mathcal{C})$ have be found 
in [23], and $\mathcal{D}$ is still known explicitly; specifically we have

$$
\left\{\begin{array}{l}
\mathcal{B}_{\mathrm{Neu}} \simeq \frac{1}{(1-\varphi)} \frac{e}{h}-\frac{2}{\pi} \log \left(\cos \frac{\pi \varphi}{2}\right), \\
\mathcal{C}_{\mathrm{Neu}} \simeq \frac{\pi}{8}(1-\varphi)^{2}\left(1-e^{-\frac{8 e}{(1-\varphi) \pi h}}\right), \\
\mathcal{D}_{\mathrm{Neu}}=(1-\varphi) \frac{e}{h}
\end{array}\right.
$$

The variations of $\left(\mathcal{B}_{\mathrm{Neu}}, \mathcal{C}_{\mathrm{Neu}}\right)$ against $e / h$ are reported in figure 7 (a) together with their values computed numerically; the excellent agreement justifies their use. Hence, once reported in (13), we get an explicit estimate of $\theta^{*}$ whose variations against $e / h$ and $\varphi$ are shown in figure 7(b). The actual Brewster angle $\theta^{*}$ has been computed numerically as the minimum of $\left|R_{\text {num }}\right|$ for each couple $(e / h, \varphi) ;($ and $k h=1)$ the agreement with the theoretical estimate is better than $3 \%$ on average for $e / h \in(0,2)$ and $\varphi \in(0,1)$.

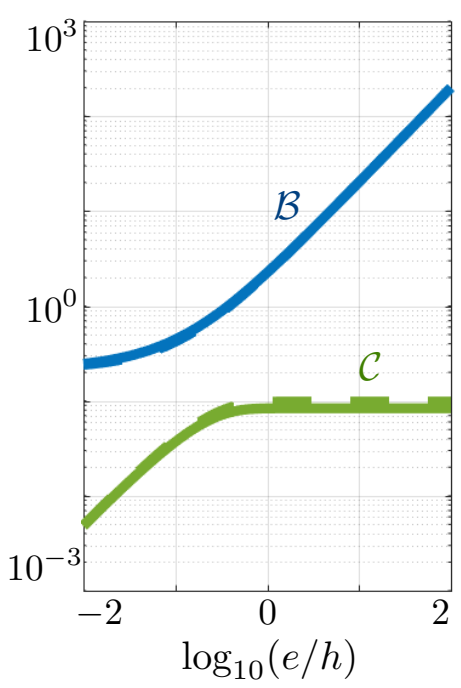

(a)

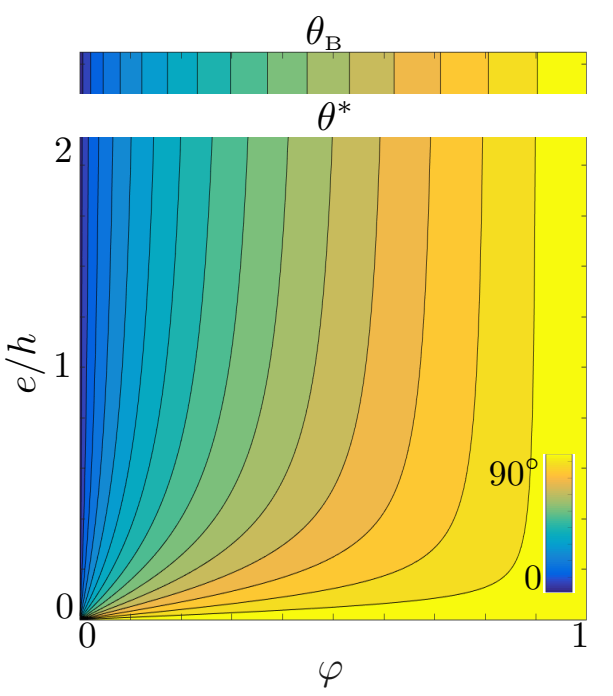

(b)

Figure 7: Limiting case of rigid films - (a) Parameters $\left(\mathcal{B}_{\mathrm{Neu}}, \mathcal{C}_{\mathrm{Neu}}\right)$ as a function of $(e / h)$ $(\varphi=0.5)$. The estimates in 16 are plotted in dashed lines. (b) Brewster angle $\theta^{*}$ against $\varphi$ and $e / h$ from 13 along with (16). The classical Brewster angle $\theta_{\mathrm{B}}=\operatorname{acos}(1-\varphi)$ is reported for comparison.

In particular, from (16), we get simplified expressions of $\theta^{*}$ in the limits of thin and thick films

$$
\left\{\begin{array}{l}
\text { for thin films, } \cos \theta^{*}=\sqrt{\frac{-2}{\log \left(\cos \frac{\pi \varphi}{2}\right)}} \frac{e}{h} \rightarrow 0, \\
\text { for thick films, } \cos \theta^{*}=(1-\varphi),
\end{array}\right.
$$


and as we have found for sound-penetrable films, the Brewster angle for thick films coincides with $\theta_{\mathrm{B}}$ given by the classical effective medium model see e.g. [5, 17. Next by continuity, it follows that perfect transmission is always possible with a Brewster angle decreasing when $e / h$ decreases, a fact observed in figure 2 (c).

The ability of the models to reproduce the scattering properties of the soundhard films is illustrated in the figures 8 and 9 . As for the case of sound-penetrable films, the actual spectrum is well reproduced by the effective interface model for thin films, here for $k e \in\left(10^{-1}-1\right)$. Conversely, for thick films, the effective medium model is more accurate; in particular it recovers the Fabry-Perot resonances occurring at $k e=n \pi$ (since $K=k$ in $(7)$ ) except in the vicinity of the intersection of two perfect transmissions; this is attributable to a deeper excitation of the evanescent field in these regions which would require to conduct the model at higher orders (see Appendix C). It is worth noting that it is more difficult in the present case to define a region of transition due to the occurence of Fabry-Perot resonances at relatively low $k e$-value. The overall error between $R_{\text {num }}$ and interface model is $7 \%$ for $k e \in\left(10^{-1}-1\right)$ and $70 \%$ for $k e \in(1-10)$; for the effective medium model, it is $40 \%$ for $k e \in\left(10^{-1}-1\right)$ and it remains as large as $20 \%$ for $k e \in(1-10)$; for the effective interface model, $(\mathcal{B}, \mathcal{C})$ have been calculated using 16 .

(a) Numerics

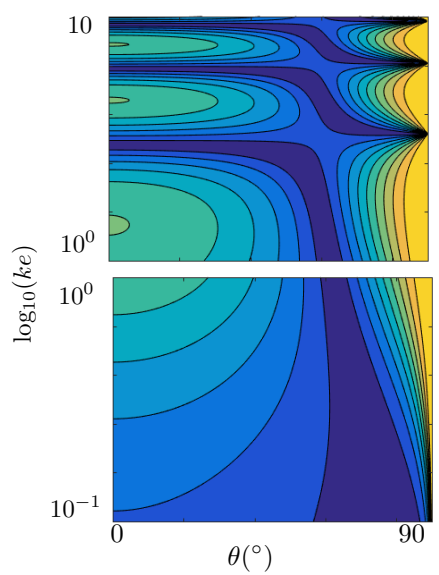

(b) effective medium model
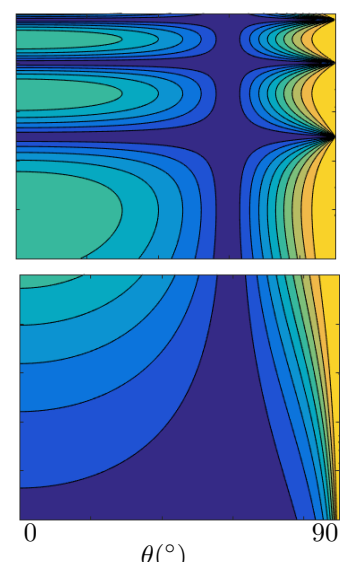

(c) effective interface model
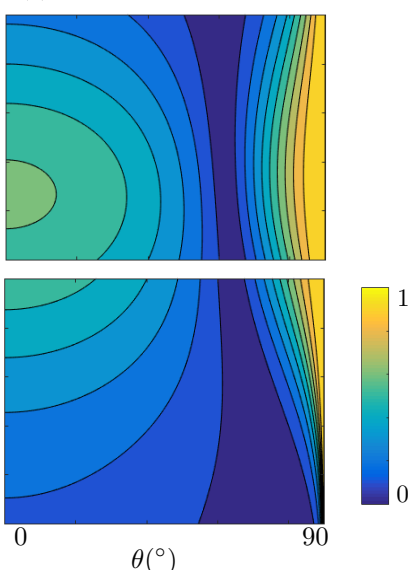

Figure 8: Reflection $|R|$ against $\theta$ and $\log _{10}(k e)$ for a perforated rigid film $(k h=1)$. Same representation as in figure 3

Eventually, typical profiles for a thin film are reported in figure 9 which illustrates the accuracy of the effective interface model. As in the figure 4 we also report in the inset the variations of $\theta^{*}$ from $90^{\circ}$ to $\operatorname{acos}(1-\varphi)$ when increasing $e / h$, in agreement with (17). For intermediate values of $e / h$, the ability of our expression (13) along with 16 to predict $\theta^{*}$ is excellent; in the present case, $\theta^{*}$ is explicit for any film thickness. 


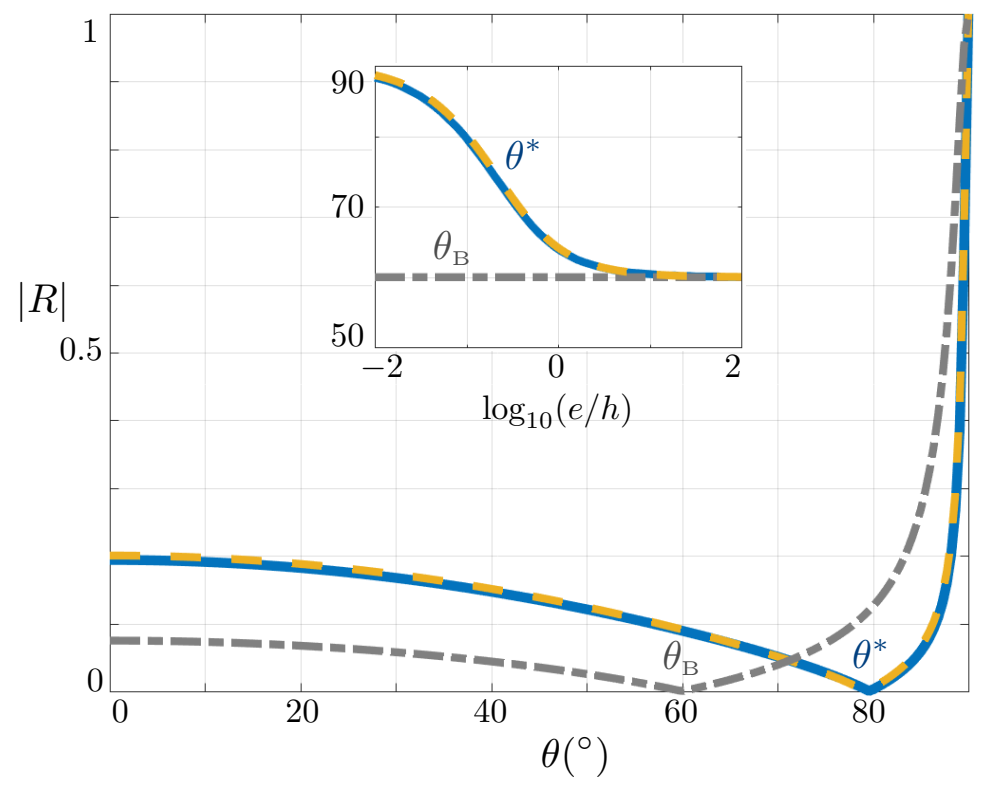

Figure 9: Reflection $|R|$ against $\theta$ for a perforated rigid film, $e / h=0.1(\varphi=0.5$ and $k h=1)$. Same representation as in figure 4

\section{Conclusion}

We have studied the scattering properties of perforated films with a focus on thin films. The conditions under which perfect transmissions are possible are modified when ultrathin devices are considered; in particular an extraordinary transmission observed for a thick film can disappear when reducing its thickness, and the reverse situation is possible as illustrated at the begining of this paper in figure 2. In all cases, a shift in the Brewster incidence $\theta^{*}$ has been exhibited : for film materials lighter than that of the surrounding matrix, $\theta^{*}$ increases with the thickness up to $\theta_{\mathrm{B}}$, for film material heavier, $\theta^{*}$ decreases with the thickness up to $\theta_{\mathrm{B}}$. The case of perforated sound-rigid films has been shown to be a limiting case of the sound-penetrable ones for a film material infinitely heavy; in this case, perfect transmission is always possible which is of particular interest since a perforated plate obtained for vanishing thickness remains very efficient to block the wave.

These features, and more generally the scattering properties of thin films, are accurately reproduced by an effective interface model which aims to capture the boundary layer effects largely dominant compared to that of wave propagation. Beyond the geometrical or compositional effects of the perforations, the thickness of the films offers a degree of freedom to tune its properties. Besides, it is a parameter of practical importance since it measures the compactness of the structure. With regards to the design of ultrathin devices, such alternative models are of particular interest since they have their range of validity which 
complements that of the classical effective medium theory.

[1] S. G. Rodrigo, F. de León-Pérez and L. Martín-Moreno, Proceedings of the IEEE, 104(12), 2288-2306 (2016).

[2] T.W. Ebbesen, H.J. Lezec, H.F. Ghaemi, T. Thio and P.A. Wolff, Nature 391667 (1998).

[3] M.H. Lu, X.K. Liu, L. Feng, J. Li, C.P. Huang, Y.F. Chen, T.Y. Zhu, S.N. Zhu and N.B. Ming, Phys. Rev. Lett., 99(17), 174301 (2007).

[4] J. Christensen, L. Martin-Moreno and F.J. Garcia-Vidal,Phys. Rev. Lett., 101(1), 014301 (2008).

[5] X.R. Huang, R.W. Peng and R.H. Fan, Phys. Rev. Lett., 105(24), 243901 (2010).

[6] G. D'Aguanno, K.Q. Le, R. Trimm, A. Alù, N. Mattiucci, A.D. Mathias, S. Aközbek and M.J. Bloemer, Scientific reports, 2, 340 (2012).

[7] A.N. Norris and X. Su, Comptes Rendus Mécanique, 343(12), 622-634 (2015).

[8] M. Edalatipour, A. Khavasi and K. Mehrany, JOSA B, 32(6), 1202-1207 (2015).

[9] P. Wei, F. Liu, Z. Liang, Y. Xu, S.T. Chu and J. Li, EPL (Europhysics Letters), 109(1), 14004 (2015).

[10] P. Peng, B. Xiao and Y. Wu, Phys. Lett. A, 378(45), 3389-3392 (2014).

[11] A. Maurel, S. Félix and J.-F. Mercier, Phys. Rev. B, 88(11), 115416 (2013).

[12] A. Akarid, A. Ourir, A. Maurel, S. Félix and J.-F. Mercier, Optics Lett., 39(13), 3752-3755 (2014).

[13] T.K. Nguyen, P.T. Dang, I. Park and K.Q. Le JOSA B, 34(3), 583-589 (2017).

[14] M. Molerón, M. Serra-Garcia and C. Daraio, New J. Phys., 18(3), 033003 (2016).

[15] G. D’Aguanno, K. Q. Le, R. Trimm, A. Alù, N. Mattiucci, A. D. Mathias, N. Akozbek and M. J. Bloemer, Scientific reports, 2, 340 (2012).

[16] D.-X. Qi, R.-H. Fan, R.-W. Peng, X.-R. Huang, M.-H. Lu, X. Ni, Q. Hu, and M. Wang, Appl. Phys. Lett. 101, 061912 (2012).

[17] A. Alù, G. D'Aguanno, N. Mattiucci, and M. J. Bloemer, Phys. Rev. Lett. 106, $123902(2011)$. 
[18] H.H. Sheinfux, I. Kaminer, Y. Plotnik, G. Bartal and M. Segev, Phys. Rev. Lett., 113(24), 243901 (2014).

[19] A. Maurel and J.-J. Marigo, Phys. Rev. B, 98(2), 024306 (2018).

[20] B. Delourme, K. Schmidt and A. Semin, Asymptotic Analysis, 97(3-4), 211-264 (2016).

[21] J.-J. Marigo and A. Maurel, In Handbook of metamaterials properties (eds R.V. Craster, S. Guenneau), vol. 2, ch. 12, pp. 599-644. Singapore: World Scientific Publishing Company.

[22] J.-J. Marigo, A. Maurel, K. Pham and A. Sbitti, J. Elas., 128(2), 265-289 (2017).

[23] J.-J. Marigo and A. Maurel, J. Acoust. Soc. Am., 140(1), 260-273 (2016).

[24] J.-F. Mercier, M.L. Cordero, S. Felix, A. Ourir and A. Maurel, P. R. S. A: Mathematical, Physical and Engineering Sciences, 471(2182), 20150472 (2015).

[25] J.-J. Marigo and A. Maurel, SIAM J. Appl. Math., 77(2), 721-743 (2017).

[26] J.-J. Marigo and A. Maurel, World Scientific Handbook of Metamaterials and Plasmonics Volume 2: Elastic, Acoustic, and Seismic Metamaterials, 2017.

\section{Appendix A. Numerical resolution}

The numerical resolution of the actual problem is performed using multimodal methods. For sound-rigid films, the method has been described in [26] and we give below the adaptation of the method for sound-penetrable films. We consider the expansion of the solution onto the basis of transverse functions $Q_{n}(x)=e^{i \beta_{n} x} \sqrt{h}$ with $\beta_{n}=k \sin \theta+2 n \pi / h$ ( $n$ integer) satisfying the condition of pseudo-periodicity for an incident wave of the form $e^{i k(\sin \theta x+\cos \theta y)}$, hence

$$
p(x, y)=p_{n}(y) Q_{n}(x),
$$

where repeated indices mean summation. In the matrix $y \notin(0, e)$ the evolution equations of the modal coefficients $p_{n}$ are not coupled and they satisfy $p_{n}^{\prime \prime}+$ $k_{n}^{2} p_{n}=0$. Hence they can be written as

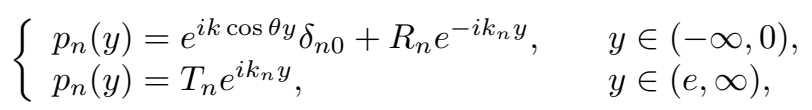

with $k_{n}=\sqrt{k^{2}-\beta_{n}^{2}}$. For $y \in(0, e)$, the heterogeneity of the medium in the transverse direction induces a coupling of the modal components $p_{n}$ and the problem then read

$$
\left\{\begin{array}{l}
\mathrm{L}_{m n} p_{n}^{\prime \prime}+\mathrm{M}_{m n} p_{n}=0, \\
\mathrm{~L}_{m n}=\mathrm{f}_{m n}\left(\rho_{\mathrm{m}} / \rho_{\mathrm{i}}\right), \quad \mathrm{M}_{m n}=k^{2} \mathrm{f}_{m n}\left(\chi_{\mathrm{i}} / \chi_{\mathrm{m}}\right)-\beta_{m} \beta_{n} \mathrm{f}_{m n}\left(\rho_{\mathrm{m}} / \rho_{\mathrm{i}}\right),
\end{array}\right.
$$


where we have defined $\mathrm{f}_{m n}(c)=\delta_{m n}+(c-1) \varphi \operatorname{sinc}(\pi(m-n) \varphi)$. It is solved using the eigensolutions of $\mathrm{N}=\mathrm{L}^{-1} \mathrm{M}=\mathrm{PKP}^{-1}$ with $\mathrm{K}$ the diagonal matrix of the eigenvalues $K_{n}^{2}$. The eigenmodes are $P_{m}=\mathrm{P}_{m n}^{-1} p_{n}=A_{n} e^{i K_{m} y}+B_{n} e^{-i K_{m}(y-e)}$ and eventually

$$
p_{n}(y)=\mathrm{P}_{n m}\left(A_{m} e^{i K_{m} y}+B_{m} e^{-i K_{m}(y-e)}\right), \quad y \in(0, e) .
$$

Next, the continuities of the pressure and of the normal velocity at $x=0$ and $x=$ $e$ can be accounted for to get the unknown complex amplitudes $\left(R_{n}, T_{n}, A_{n}, B_{n}\right)$. This is done on average, namely defining $\mathcal{Y}=\{|x| \in(0, h / 2)\}, \mathcal{Y}_{\mathrm{i}}=\{|x| \in$ $(0, \varphi h / 2)\}$ and $\mathcal{Y}_{\mathrm{m}}=\{|x| \in(\varphi h / 2, h / 2)\}$, we use that

$$
\left\{\begin{array}{l}
\int_{\mathcal{Y}} p\left(x, 0^{-}\right) \overline{Q_{m}}(x) \mathrm{d} x=\int_{\mathcal{Y}} p\left(x, 0^{+}\right) \overline{Q_{m}}(x) \mathrm{d} x, \\
\int_{\mathcal{Y}} \partial_{y} p\left(x, 0^{-}\right) \overline{Q_{m}}(x) \mathrm{d} x=\int_{\mathcal{Y}_{\mathrm{m}}} \partial_{y} p\left(x, 0^{+}\right) \overline{Q_{m}}(x) \mathrm{d} x+\frac{\rho_{\mathrm{m}}}{\rho_{\mathrm{i}}} \int_{\mathcal{Y}_{\mathrm{i}}} \partial_{y} p\left(x, 0^{+}\right) \overline{Q_{m}}(x) \mathrm{d} x,
\end{array}\right.
$$

and the same at $x=e$. The resulting system reads

$$
\left(\begin{array}{cccc}
-\mathrm{I} & \mathrm{P} & \mathrm{PE} & 0 \\
\mathrm{k} & \mathrm{H} & -\mathrm{HE} & 0 \\
0 & \mathrm{PE} & \mathrm{P} & -\mathrm{I} \\
0 & \mathrm{HE} & -\mathrm{H} & -\mathrm{k}
\end{array}\right)\left(\begin{array}{c}
\mathbf{R} \\
\mathbf{A} \\
\mathbf{B} \\
\mathbf{T}
\end{array}\right)=\left(\begin{array}{c}
\mathbf{S}_{1} \\
\mathbf{S}_{2} \\
\mathbf{0} \\
\mathbf{0}
\end{array}\right)
$$

where $\mathrm{I}$ is the identity matrix, $\mathrm{E}=e^{i \mathrm{~K} e}, \mathrm{k}_{m n}=k_{m} \delta_{m n}, \mathrm{H}_{m n}=\mathrm{f}_{m p}\left(\rho_{\mathrm{m}} / \rho_{\mathrm{i}}\right) \mathrm{P}_{p n} \mathrm{~K}_{n}$. The vectors $(\mathbf{R}, \mathbf{T}, \mathbf{A}, \mathbf{B})$ are the column vectors of the coefficients $\left(R_{n}, T_{n}, A_{n}, B_{n}\right)$ in the truncated series $n \in(-N, N)$ and the source terms are $S_{1, n}=\delta_{n 0}$, $S_{2, n}=k \cos \theta \delta_{n 0}$. It is now sufficient to invert the above system to get the unknown amplitudes in A.2 and A.4 and thus the whole solution in A.1).

\section{Appendix B. Effective parameters $(\mathcal{B}, \mathcal{C})$ and their bounds}

In the jumps of pressure and normal velocity, three so-called interface parameters among which $(\mathcal{B}, \mathcal{C})$ have to be determined numerically. They are defined as

$$
\left\{\begin{array}{l}
\mathcal{B} \equiv \frac{e}{h}+\left(\lim _{y_{1} \rightarrow+\infty} Q_{\mathrm{y}}-\lim _{y_{1} \rightarrow-\infty} Q_{\mathrm{y}}\right), \\
\mathcal{C} \equiv \frac{e}{h}\left(\varphi \frac{\rho_{\mathrm{m}}}{\rho_{\mathrm{i}}}+1-\varphi\right)+\int_{\Omega_{\infty}} \mathrm{d} \boldsymbol{\xi} \frac{\rho_{\mathrm{m}}}{\rho(\boldsymbol{\xi})} \frac{\partial Q_{\mathrm{x}}}{\partial \xi_{x}}(\boldsymbol{\xi}),
\end{array}\right.
$$

with $\boldsymbol{\xi}=\left(\xi_{x}, \xi_{y}\right)=(x, y) / h$ a rescaled coordinate and $\varphi=\mathcal{S}_{\mathrm{i}} / h^{2}$ where $\mathcal{S}_{\mathrm{i}}$ is the surface of the perforated film. The parameter $\mathcal{D}$ is given explicitly and $(\mathcal{B}, \mathcal{C})$ are deduced from static problems set on $\left(Q_{\mathrm{x}}, Q_{\mathrm{y}}\right)$, and satisfying for $\alpha=x, y$

It is worth noting that these problems have several advantages (i) they are static, (ii) they depend on the contrast in mass density only, the contrast in the sound speed being encapsulated explicitly in $\chi_{\mathrm{r}}$, (iii) they are set in a nondimensional geometry (with a reference length $h$ ). As such $(\mathcal{B}, \mathcal{C})$ hold for a large 


$$
\left\{\begin{array}{l}
\operatorname{div}\left[\frac{\rho_{\mathrm{m}}}{\rho} \nabla\left(Q_{\alpha}+\xi_{\alpha}\right)\right]=0 \\
Q_{\alpha} \\
\frac{\rho_{\mathrm{m}}}{\rho} \partial_{n}\left(Q_{\alpha}+\xi_{\alpha}\right) \\
\lim _{\xi_{y} \rightarrow \pm \infty} \nabla Q_{\alpha}=\mathbf{0}
\end{array}\right.
$$

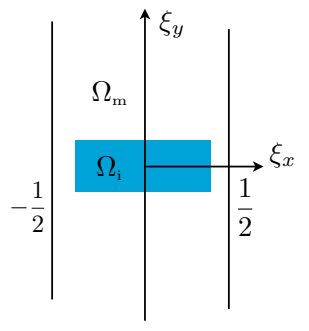

Figure B.10: Elementary problems on $\left(Q_{x}, Q_{y}\right)$ set in $\Omega=\Omega_{\mathrm{i}} \cup \Omega_{\mathrm{m}}=(-1 / 2,1 / 2) \times(-\infty, \infty)$ used to calculate $(\mathcal{B}, \mathcal{C})$, Eqs. B.1.

family of settings; besides, once they are determined, the effective problem can be solved in the transient as in the harmonic regime for any kind of source and any radiation or boundary conditions.

\section{Appendix B.1. Upper bound for $\mathcal{B}$}

Determining an upper bound for $\mathcal{B}$ requires to invoke the Thomson principle (a similar calculation can be found in [22] for the determination of the upper bound of $\mathcal{C}$ ). It is based on the equivalence between the problem on $Q_{\mathrm{y}}$ and the problem of energy minimization for $\boldsymbol{U}=\left(\rho_{\mathrm{m}} / \rho\right) \boldsymbol{\nabla}\left(Q_{\mathrm{y}}+\xi_{y}\right)$ which reads

$$
\begin{aligned}
& E(\boldsymbol{U}) \leq E(\tilde{\boldsymbol{U}}), \\
& \text { with } E^{*}(\tilde{\boldsymbol{U}})=\frac{1}{2} \int_{\Omega} \mathrm{d} \boldsymbol{\xi} \frac{\rho}{\rho_{\mathrm{m}}}\left|\tilde{\boldsymbol{U}}-\frac{\rho_{\mathrm{m}}}{\rho} \boldsymbol{e}_{y}\right|^{2},
\end{aligned}
$$

for any admissible field $\tilde{\boldsymbol{U}}$ with $\tilde{\boldsymbol{U}} \cdot \mathbf{n}$ continuous, $\xi_{x}$-periodic in $\Omega$ and satisfying $\operatorname{div} \tilde{\boldsymbol{U}}=0, \tilde{\boldsymbol{U}} \rightarrow \boldsymbol{e}_{y}$ for $\xi_{y} \rightarrow \pm \infty$. We start by linking $E(\boldsymbol{U})=$ $\frac{1}{2} \int_{\Omega} \mathrm{d} \boldsymbol{\xi} \frac{\rho_{\mathrm{m}}}{\rho}\left|\nabla Q_{\mathrm{y}}\right|^{2}$ to $\mathcal{B}$; we use that $0=\int_{\Omega} \mathrm{d} \boldsymbol{\xi}\left(Q_{\mathrm{y}}-\xi_{y}\right) \operatorname{div}\left[\frac{\rho_{\mathrm{m}}}{\rho} \boldsymbol{\nabla}\left(Q_{\mathrm{y}}+\xi_{\mathrm{y}}\right)\right]$, from which making use of the boundary conditions

$$
\int_{\Omega} \mathrm{d} \boldsymbol{\xi} \frac{\rho_{\mathrm{m}}}{\rho}\left|\nabla Q_{\mathrm{y}}\right|^{2}=\int_{\Omega} \mathrm{d} \boldsymbol{\xi} \frac{\rho_{\mathrm{m}}}{\rho}+\lim _{\xi_{m} \rightarrow+\infty}\left[Q_{\mathrm{y}}-\xi_{y}\right]_{-\xi_{m}}^{+\xi_{\mathrm{m}}} .
$$

Both terms on the right hand side diverge in the above expression, but not their sum; specifically, we have

$$
\left\{\begin{array}{l}
\int_{\Omega} \mathrm{d} \boldsymbol{\xi} \frac{\rho_{\mathrm{m}}}{\rho}=2 \lim _{\xi_{m} \rightarrow+\infty} \xi_{m}-\varphi\left(1-\frac{\rho_{\mathrm{m}}}{\rho_{\mathrm{i}}}\right) \\
\lim _{\xi_{m} \rightarrow+\infty}\left[Q_{\mathrm{y}}-\xi_{y}\right]_{-\xi_{m}}^{+\xi_{m}}=\left(\mathcal{B}^{+}-\mathcal{B}^{-}\right)-2 \lim _{\xi_{m} \rightarrow+\infty} \xi_{m}
\end{array}\right.
$$

where $\mathcal{B}^{ \pm}=\lim _{y_{1} \rightarrow \pm \infty} Q_{\mathrm{y}}$. Hence

$$
E(\boldsymbol{U})=\frac{1}{2}\left[\left(\mathcal{B}^{+}-\mathcal{B}^{-}\right)-\varphi \frac{e}{h}\left(1-\frac{\rho_{\mathrm{m}}}{\rho_{\mathrm{i}}}\right)\right] .
$$


It is now sufficient to choose the simple admissible field $\tilde{\boldsymbol{U}}=\boldsymbol{e}_{y}$, which provides $E(\tilde{\boldsymbol{U}})=\frac{\varphi e}{2 h} \frac{\rho_{\mathrm{i}}}{\rho_{\mathrm{m}}}\left(1-\frac{\rho_{\mathrm{m}}}{\rho_{\mathrm{i}}}\right)^{2}$ and to invoke $\mathrm{B} .2$ to get that $\mathcal{B}=\left(\mathcal{B}^{+}-\mathcal{B}^{-}\right)+\frac{e}{h}$ in (B.1) satisfies

$$
\mathcal{B} \leq \frac{e}{h}\left(\varphi \frac{\rho_{\mathrm{i}}}{\rho_{\mathrm{m}}}+1-\varphi\right) .
$$

Appendix B.2. Upper bound for $\mathcal{C}$

The upper bound on $\mathcal{C}$ is straightforward. It is sufficient to use that

$$
\int_{\Omega} \mathrm{d} \boldsymbol{\xi} Q_{\mathrm{x}} \operatorname{div}\left[\frac{\rho_{\mathrm{m}}}{\rho} \nabla\left(Q_{\mathrm{x}}+\xi_{x}\right)\right]=0,
$$

from which

$$
\int_{\Omega} \mathrm{d} \boldsymbol{\xi} \frac{\rho_{\mathrm{m}}}{\rho} \frac{\partial Q_{\mathrm{x}}}{\partial \xi_{x}}(\boldsymbol{\xi})=-\int_{\Omega} \mathrm{d} \boldsymbol{\xi} \frac{\rho_{\mathrm{m}}}{\rho}\left|\nabla Q_{\mathrm{x}}\right|^{2} \leq 0,
$$

where we used the boundary conditions on $Q_{\mathrm{x}}$. With $\mathcal{C}$ defined in $\mathrm{B} .1$, we get the upper bound

$$
\mathcal{C} \leq \frac{e}{h}\left(\varphi \frac{\rho_{\mathrm{m}}}{\rho_{\mathrm{i}}}+1-\varphi\right) .
$$

\section{Appendix C. Remark on the effective medium model at higher order}

In the comment of the figure 8 , we have stress that the relatively bad agreement between the actual spectrum for $k e \in(1-10)$ and that predicted by the classical effective medium model was attributable to the effect of the evanescent field. In this appendix, we justify this statement. To begin with, we report in figure C.11 the same figures than in figure 8 but with the actual spectrum (a) calculated using a monomodal version of the multimodal method; this means that the numerics is performed with the propagating mode only. Doing so, the evanescent field is neglected. It results a spectrum (a) which ressembles that given by the effective medium model (b) with agreement better than $2 \%$ on the whole range $k e \in\left(10^{-1}-10\right)$. This confirms that the effect of the evanescent field are dominant for $k e \in\left(10^{-1}-1\right)$ and significant for $k e \in(1-10)$.

This failure can be remediated by pursuing the asymptotic analysis to higher order which means by improving the model. This has been done in 25] and we report below the result to illustrate the resulting gain for thick films. The figure C.12 reports the same figures (a) and (c) than in figure 8 but the panel (b) shows now the spectrum obtained with the effective medium model conducted at high order. The improvement in the prediction it is very significantly increased for $k e \in(1-10)$, with an error reduced to $0.5 \%$. Expectedly, it remains high for $k e \in\left(10^{-1}-1\right)$, with an error of $20 \%$. 


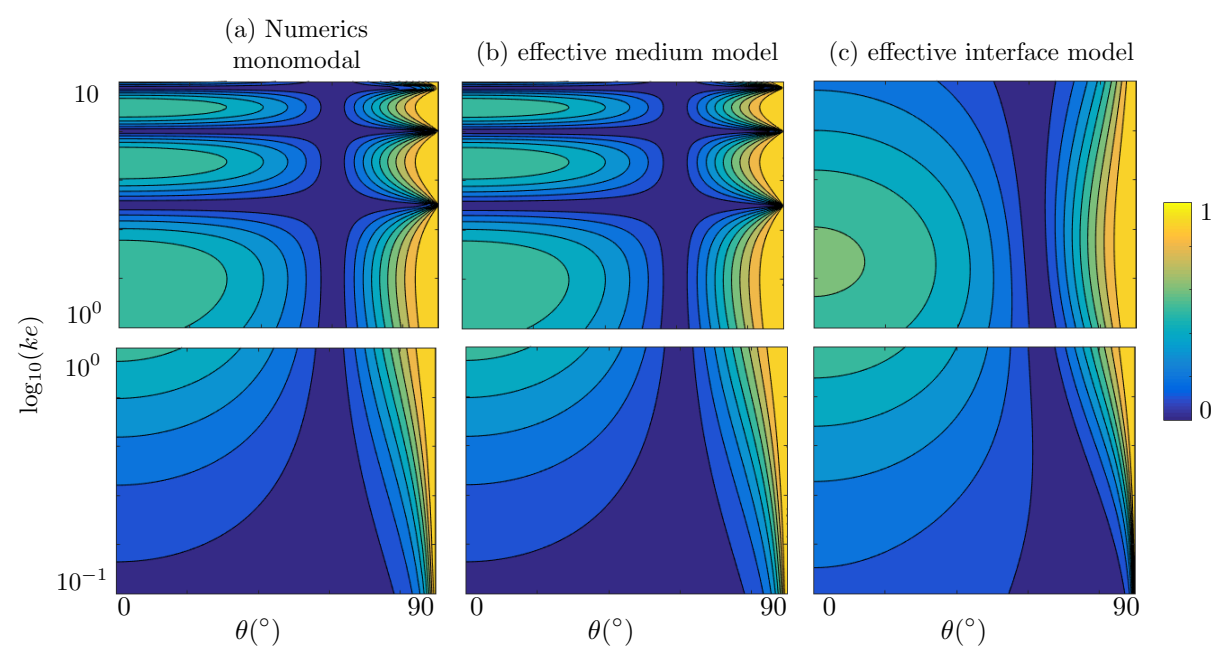

Figure C.11: Same representation as in figure 8 with in (a) the result of the monomodal version of the numerics, hence omitting the evanescent field.

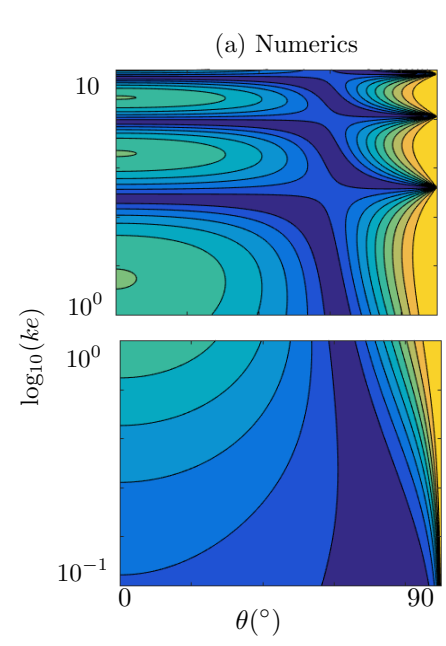

(b) effective medium model

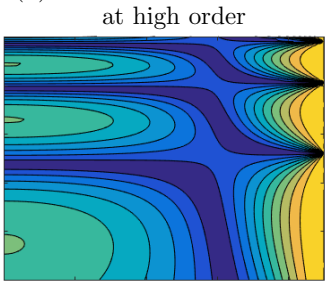

(c) effective interface model
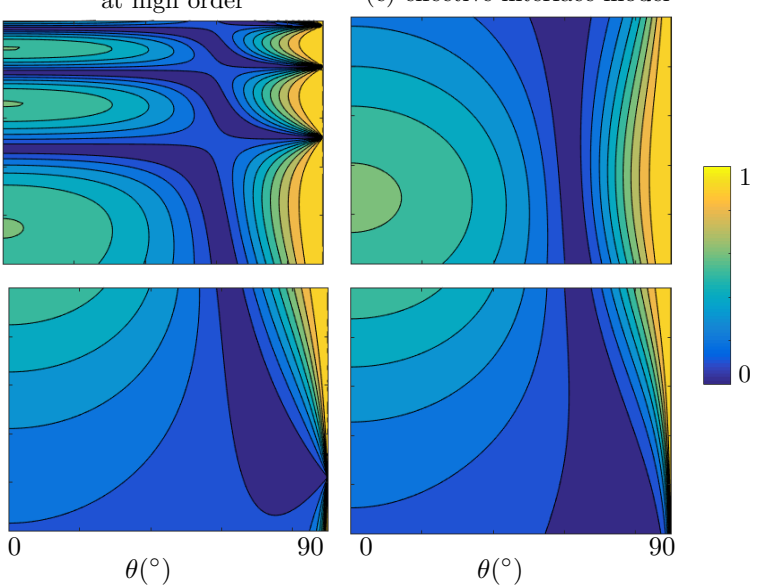

Figure C.12: Same representation as in figure 8 with in (b) the result of the effective medium model at the higher order 1 , from [25]. 